\title{
Influence of the Injection Speeds on the Discharge Characteristics and Minimization of Delay Time in a Pseudospark Discharge
}

\author{
S.O. Leathem ${ }^{*}, 1$, P. Stoltz ${ }^{1}$, P. Messmer ${ }^{1}$, D.L. Bruhwiler ${ }^{1}$ and J.-L. Cambier ${ }^{2}$ \\ ${ }^{1}$ Tech-X Corp.,5621 Arapahoe Ave., Suite A, Boulder, CO 80303, USA \\ ${ }^{2}$ Air Force Research Laboratory, AFRL/PRSA, Edwards AFB, CA 93524, USA
}

\begin{abstract}
The study put forth demonstrates that the seed electrons average kinetic energy influences the discharge characteristics making it possible to maximize the rate of development of the virtual anode in a pseudospark, with a suitable choice of the neutral gas pressure as determined by the seed's average injection speed. This investigation also brings to light two distinct operating regimes; (1) mid-energy, where electron-impact ionization energy losses result in a decrease in the cross-section as the electrons travel downstream and (2) high-energy, where, in contrast, the ionization cross-section increases. In the latter case, both the fastest delay time and the neutral gas pressure producing this value have linear dependencies on the seed electrons energy resulting in a constant value of their product over the different injection speeds. The discharge is seeded by injecting a current pulse for a period of one nanosecond along the axis from the hollow cathode cavity back wall over a range of mean speeds corresponding to 100 to $900 \mathrm{~V}$ accelerations; the initial electric field is insufficient to enhance ionization throughout most of the hollow cathode backspace. Data is obtained through computer simulation using the two-dimensional kinetic plasma code OOPIC Pro. 51.50.+v, 52.75.Kq, 52.80.Tn.
\end{abstract}

Keywords: Pseudospark, delay time, breakdown, high current switch.

\section{INTRODUCTION}

The pseudospark [1-6] is a low pressure gas discharge comprised of hollow cathode and anode cavities each covered by an electrode with a hole in the centre. This device is capable of producing a rapid current rise up to $10^{12} \mathrm{~A} / \mathrm{s}$ [1] with current densities $>10^{6} \mathrm{~A} / \mathrm{cm}[2,4]$; typical rise times are $\sim 10 \mathrm{~ns}$.

Commonly, psuedosparks [7-12] are used as a high voltage, high current switch. Therefore, it is desirable to achieve a low delay time between the trigger and breakdown in the main gap. The physics of pseudospark operation can be complex and several characteristic phases of development have been identified by various authors. Although the nomenclature of such phases can be varied, it is generally agreed [13-15] that the discharge is characterized by space-charge build-up, followed by rapid ionization avalanche and electron beam formation, and finally a super-emissive ion state. The physical model for the latter phase (also called "super-dense glow") is not yet well understood, albeit likely to involve a self-sustained sputtering [16], and is not being modelled here; our attention is focused on the triggering and avalanche processes, which we identify as the (a) prebreakdown and; b) breakdown phase. During the pre-breakdown phase electrons generated through ionization are accelerated to the anode leaving behind a region of net positive charge called the virtual anode. This is followed by an increasing plasma particle generation rate inside the virtual anode which leads to high plasma densities and the slow expansion of the virtual anode upstream in the breakdown phase. It is during this phase that an electron beam forms along the axis.

*Address correspondence to this author at E-mail: soleathem@gmail.com
The effect of injecting a current pulse on the discharge breakdown characteristics in other configurations has received previous attention [17-20]. When a current pulse is used to seed the device the minimum voltage needed across the gap to achieve breakdown decreases [18-20]. Cooley and Choueiri [19] have shown that this is due to the enhancement of the electric field resulting from the space charge generated by the current. Additionally, experiments have shown that the number [8] of seed electrons needed to initiate a discharge is of the order of $10^{9}$ to $10^{10}$.

A previous investigation [17] of the pseudospark discharge, with variable hollow cathode dimensions, showed that, in general, the magnitude of the peak electron current grows as the mean seed injection energy increases.

A study is presented which illustrates the influence of the mean seed injection velocity on the delay time for a wide range of neutral gas pressures. Fixing the geometry, the dimensions ensure the electric field across the anode-cathode gap does not enhance ionization throughout most of the hollow cathode interior. Additionally, the investigation further clarifies the variation of the general discharge properties according to which energy range the injection electrons fall into; medium energy, where the electron impact ionization cross-section decreases after collisional energy losses or high energy, where the electron impact ionization cross-section increases after collisional energy losses. In section II there is a description of the physical model employed in the study and the code used to generate the simulations, OOPIC Pro. This is followed by a discussion of the results in section III. 


\section{THE MODEL}

\subsection{The Simulation Framework}

The numerical investigation is performed using twodimensional electrostatic kinetic particle-in-cell simulations generated by OOPIC Pro [21]. This code accounts for both electron and ion energy dependent collisions (see section 2.2) and electron and ion impact secondary electrons from the surfaces.

The simulation parameters such as the cell-size, time-step and particle-weighting have been selected to minimize the impact of fluctuations and grid heating. Each parameter has been decreased to a point where successive reductions produce similar physical results and the largest values with consistent outcomes have been selected to minimize computing time.

An illustration of the geometry used is shown in Fig. (1). The model employs a fixed hollow cavity height $Y_{\max }=50$ mm, anode-cathode gap $d=5 \mathrm{~mm}$, depth $D=25 \mathrm{~mm}$, cathode thickness $w=3 \mathrm{~mm}$ and hole height $h=4 \mathrm{~mm}$.

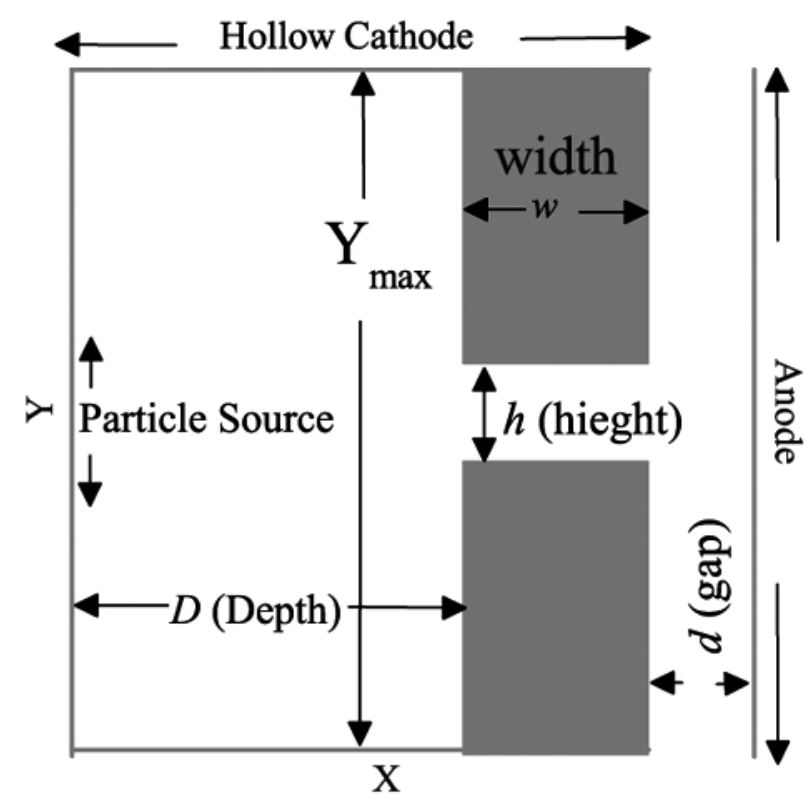

Fig. (1). The model setup, where PS indicates the electron seed injection location.

The model ignores the details of the particle source and sends a beam of electrons with a predefined drift velocity and temperature into the hollow cathode cavity. A total of 1 $\mathrm{x} 10^{10}$ seed electrons are injected at the centre of the hollow cavity's back wall over a region that extends the same value as the hole height (see Fig. 1) for a duration of one nanosecond. This interval of time is less than that taken to form a discharge in the prebreakdown stage for all the cases studied. The current pulse injects electrons with a Maxwellian velocity distribution at a temperature of $2.5 \mathrm{eV}$ and a mean velocity normal to the wall corresponding to $100,300,500,700$ or $900 \mathrm{~V}$ accelerations; the peak in the ionization cross-section occurs at $67 \mathrm{eV}$. For a given mean injection velocity simulations are run over a range of neutral gas pressures extending from 0.1275 to 1.8 torr.
Common to all simulations is the use of Argon gas, an anode voltage of $10 \mathrm{kV}$, a grounded cathode and the inclusion of secondary electrons. The secondary electrons are due to electron and ion impact of the surfaces, where molybdenum is used for the cavity and steel for the cathode electrode. A constant secondary coefficient is used for ion impact, whereas a Vaughan based secondary production model that takes into account energy and angular dependencies is used for the electrons.

This study employs a wide range of neutral gas pressures. At the lower end of the scale, the neutral gas pressure is $\mathrm{P}=$ 0.18 torr and the ionization mean-free-path at the peak in the cross-section is $5.88 \mathrm{~mm}$; at this same value of electron energy the elastic mean-free-path is much smaller at 1.93 $\mathrm{mm}$. These paths grow substantially with the electron energy, $U$, where for $U=1000 \mathrm{eV}$ the ionization and elastic mean-free-paths are 23.7 and $11.3 \mathrm{~mm}$, respectively. Both of these mean-free-paths are less than the hollow cathode length. These collisions also play a substantial role in the two-dimensional development of the discharge by scattering electrons normal to the injection direction.

The system modelled continuously evolves and only the first two stages of its development (prebreakdown and breakdown phases) are studied. All simulations are run up to the point when the peak electron current at the anode (disappearance of the voltage difference across the anodecathode gap) occurs.

\subsection{The Collision Model}

OOPIC Pro uses the null collision method [22] for MCC (Monte Carlo collision) treatment of electron-impact excitation and ionization (outer electron only) and for electron-neutral elastic scattering. Nonrelativistic MCC models were implemented over ten years ago [22], eventually with support for atomic Ar, Ne, He and H. More recently, fully relativistic models were implemented [23], including improved treatment of the low-energy scattering angles for both primary and secondary electrons. These more recent algorithms are presented in detail here. The relativistic aspects of these algorithms are not directly relevant to this paper, but they are integral to the algorithms and so must be discussed for the sake of completeness.

The total cross sections $\sigma(E)$, as originally implemented in OOPIC Pro for electron-neutral collisions, fall from their maximum value like $\ln (E) / E$ as the impact energy $E$ increases. This behavior is correct for $E<200 \mathrm{KeV}[23,24]$. However, relativistic effects break this scaling, leading to a minimum $[23,24]$ in $\sigma(E)$ for $E \sim 1 \mathrm{MeV}$. As $E$ increases beyond $1 \mathrm{MeV}, \sigma(E)$ grows logarithmically, until it eventually saturates at a density dependent energy (the Fermi plateau) $[25,26]$.

Reiser developed a simple fitting function for impact ionization cross sections [23], using the ionization energy and two adjustable parameters, approximately capturing both low-energy and relativistic behavior (but not the Fermi plateau). The fitting parameters for this previous work were determined largely by data for impact energies near $1 \mathrm{MeV}$, which has been published for a number of gasses [27]. We found this approach to be inaccurate for sufficiently high electron energies. 
We now describe a parametric model for the total impact ionization cross section at energies ranging from the ionization threshold energy up to $100 \mathrm{GeV}$. Many parametric models have been developed for $T<200 \mathrm{KeV}$. For example,

$$
\sigma(T, I)=\frac{1}{u I^{2}}\left[C_{1}\left(1-\frac{1}{u}\right)+C_{2}\left(1-\frac{1}{u^{2}}\right)+C_{3} \ln (u)+\frac{C_{3}}{u} \ln (u)\right],
$$

where $u=T / I$ is the kinetic energy normalized to the ionization threshold energy, and the model breaks down as the electron becomes relativistic. The relativistic rise can be captured by a function like the following:

$\sigma(T, I) \sim C_{1}\left[\frac{1}{\beta^{2}} \ln \left(C_{2} \beta^{2} \gamma^{2}\right)-1\right]$,

where $\beta=v / c$ is the normalized electron velocity and $\gamma$ is the usual relativistic factor. This simple form cannot, by itself, capture the low-energy behavior of $\sigma(T, I)$. Nor can it capture the Fermi plateau. However, the following, more complicated model can accurately match data for the lowenergy behavior and simultaneously capture the relativistic rise and subsequent saturation due to the Fermi plateau,

$$
\begin{aligned}
\sigma(T, I)= & \frac{C_{1}}{u_{r e l}}\left(1-\frac{1}{u_{r e l}}\right)+\frac{C_{2}}{u_{r e l}^{2}} \ln \left(u_{n r}\right)+\frac{C_{3}}{u_{n r}} \ln \left(u_{n r}\right) \\
& +C_{3}\left(1-\frac{1}{u_{r e l}^{2}}\right)\left[\frac{1}{u_{n r}} \ln \left(C_{3} \gamma^{2}\right)-\frac{2 I}{m_{e} c^{2}}\right]
\end{aligned}
$$

where $u_{n r}=.5 \beta^{2} m_{e} c^{2} / I$, and $u_{\text {rel }}=(\gamma-1) \mathrm{m}_{e} c^{2} / I$.

This model has been implemented in OOPIC Pro, with appropriate coefficients for $\mathrm{H}, \mathrm{Li}$ and $\mathrm{N}$. The fitting parameters for ionization were determined from Younger [23] (low energy) and Perkins et al. [23] (high energy). The fitting parameters for elastic scattering were determined from Bray, Fursa and McCarthy [23] (low energy) and Perkins et al. [28] (high energy).

The original OOPIC Pro model for the energy distribution of the secondary electrons is also nonrelativistic. Our new differential cross section has the form $\sigma(T, W, I)$, where $W$ is the kinetic energy of the ejected secondary electron. From energy conservation, the final energy of the primary electron is $T_{f}=T-W-I$. A number of parametric functions have been developed to describe the energy distribution of secondary electrons at low [23, 25] and moderately high [24] energies. For example, the binary encounter approximation is valid at low energy,

$\sigma(T, W, I) \sim\left[\frac{1}{(W+I)^{2}}+\frac{4 I}{3(W+I)^{3}}+\frac{1}{(T-W)^{2}}+\frac{4 I}{3(T-W)^{3}}-\frac{1}{(W+I)(T-W)}\right]$. [29]:

The theory for very high energies is also well established

$$
\sigma(T, W, I) \sim\left[\frac{1}{w^{2}}+\frac{1}{(T-W)^{2}}-\frac{1}{W(T-W)} \frac{m c^{2}\left(2 T+m c^{2}\right)}{\left(T+m c^{2}\right)^{2}}+\frac{1}{\left(T+m c^{2}\right)^{2}}\right] .
$$

The following, more complicated model can asymptotically capture both the low-energy and high-energy models above:

$$
\sigma(T, W, I)=\frac{\sigma(T)}{f(T, I)}\left[\begin{array}{l}
\frac{1}{(W+I)^{2}}+\frac{4 I}{3(W+I)^{3}}+\frac{4 I}{3(T-W)^{3}} \\
+\frac{1}{(T-W)^{2}}-\frac{1}{(W+I)(T-W)} \frac{m c^{2}\left(2 T+m c^{2}\right)}{\left(T+m c^{2}\right)^{2}}+\frac{1}{\left(T+m c^{2}\right)^{2}}
\end{array}\right],
$$

where $f(T, I)$ is a normalizing function that has been calculated. Given an appropriate form of $\sigma(T, I)$ for a specified atom, and the corresponding value of $I$, this model does not require any other species-dependent information.

Equation (6) defines the differential cross-section for the kinetic energies of the incident and ionized electrons, denoted by $T$ and $W$, respectively. Here, $\mathrm{I}$ is the usual ionization threshold energy, and $f(T, I)$ is a normalizing function, which is defined by the condition that integration of $\sigma(T, W, I)$ with respect to $W$, over the range $0<W<T-I$, must equal $\sigma(T, I)$.

The differential cross section, defined in Equation (6) is used to calculate the kinetic energy of the impact ionized electrons, denoted by $W$, with a physically correct distribution of values. The probability for impact ionization to occur is first calculated using the full cross section $\sigma(T, I)$. If an event is determined to occur, then $\sigma(T, W, I)$ is used to calculate $W$ for the generated electron, while the incident electron's kinetic energy is reduced from $T$ to $(T-W$ I).

Once $\mathrm{W}$ has been calculated, one can then use $T, W$ and $I$ to calculate, from the doubly differential cross section, the scattering angles of the two electrons.

In the ultra-relativistic (high-energy) limit, the cross sections can be calculated theoretically. In the low-energy limit, one can find in the literature a variety of parametric forms that accurately capture experimental data. The parametric forms used in OOPIC Pro have been chosen because they agree asymptotically with appropriate functional forms in both the high-energy and low-energy limits. The values of the various parametric coefficients are obtained through a least squares fit to data available in the literature.

Finally, one must also consider the doubly differential cross section, which in the most general case has the form $\sigma\left(T, W, \theta_{\mathrm{p}}, \theta_{\mathrm{s}}, \phi_{\mathrm{p}}, \phi_{\mathrm{s}}, I\right)$, where $\theta$ and $\phi$ are the polar and azimuthal scattering angles with respect to the initial direction of the primary electron, and the subscripts $p$ and $s$ refer to the primary and secondary electrons, respectively. In practice, the function form is much simpler than this, because the scattering is azimuthally uniform. Also, the primary and secondary angles are uncorrelated at low energy but strongly correlated at high energy. Thus, we can use the form $\sigma(T, W, \theta, I)$.

Several works discuss the angular distribution for elastic and inelastic scattering in the nonrelativistic [29-31] and relativistic regimes [26-42]. At low energy, the following form agrees well with data: 
$$
\sigma(T, W, \theta, I) \sim 1 /\left\{G_{3}(T, W, I)+\left[\cos \theta-G_{2}(T, W, I)\right]^{2}\right\},
$$

where,

$G_{2}(T, W, I)=\sqrt{(W+I) / T}$,

and

$G_{3}(T, W, I)=\alpha \sqrt{I(T-W-I) /(T W)}$,

with $\alpha \approx 0.6$ a fitting parameter. Also, there is a normalizing function - not shown - which is a function of the singly differential cross section $\sigma(T, W, I)$. At low energy, one uses the doubly differential cross section with a straightforward Monte Carlo algorithm to determine the polar scattering angle for the primary electron, given $T$ and $I$, as well as the value of $W$ obtained from the singly differential cross section. The polar scattering angle for the secondary electron is obtained in exactly the same way, only interchanging the values of $T$ and $W$. The azimuthal angles for both primary and secondary electrons are assumed to be randomly distributed between 0 and $2 \pi$.

At high energy, the ionization is essentially an e-/ecollision, and the polar scattering angle of the primary electron is given unambiguously by the equation,

$\cos \left(\theta_{p}\right)=\sqrt{\frac{W}{T} \frac{\left(T+2 m c^{2}\right)}{\left(W+2 m c^{2}\right)}}$,

while the azimuthal angle is still isotropic. In this limit, the secondary electron is scattered in a direction opposite to that of the primary electron, so that $\theta_{\mathrm{s}}=\pi / 2-\theta_{\mathrm{p}}$ and $\phi_{\mathrm{s}}=\phi_{\mathrm{p}}+\pi$.

The literature on scattering is split between the nonrelativistic and relativistic limits, with little discussion of the overlap regime. We have implemented new parametric models for the scattering due to both impact ionization and elastic collisions, which have the correct forms at both energy extremes and transition sensibly from the low energy to the high energy regime. This is accomplished by replacing the $G_{2}$ function above with the following form:

$G_{2}(T, W, I) \rightarrow \sqrt{\frac{(W+I)}{T} \frac{\left(T+2 m c^{2}\right)}{\left(W+I+2 m c^{2}\right)}}$,

and by replacing the fitting parameter with the more complicated form:

$\alpha \rightarrow 0.6\left(\frac{m c^{2}}{T+m c^{2}}\right)^{2}$,

With these changes, the behavior of the polar scattering angles are asymptotically correct in both the low- and highenergy limits. The azimuthal scattering angle of the secondary electron is chosen, depending on the values of $T$ and $W$, so as to also obtain asymptotically correct behavior.

The relevant cross sections for electron impact excitation (and associated scattering of the primary) and for elastic scattering are handled in an analogous manner.
The implementation of our parametric impact ionization model for additional elements is relatively straightforward. The most important piece is an accurate fit to the total cross section as a function of impact energy. Numerical values for impact ionization, impact excitation and elastic scattering at high energies can be obtained from an unpublished LLNL report [43], which has built into it an assumed neutral density and hence provides one data point to constrain the Fermi plateau. This report also provides results for low impact energies, but these results are not always very accurate. The appropriate coefficients for our parametric model are obtained by a least squares fit to the assembled set of tabulated experimental and numerical data. For elements with no relevant experimental data in the literature, one can use the simple model of Reiser, which is accurate to within a factor of a few for most energies of interest. Once the total cross section is known, the differential and doubly differential cross sections follow in a straightforward manner.

Unlike impact ionization, the elastic scattering formulae are quite generic - depending only on the atomic number of the neutral atoms [27] - so very little effort is required to make the implementation appropriate for essentially all mono-atomic atoms. We neglect impact ionization of ions (and impact ionization of any but the outermost electron from neutrals), because the cross sections for these processes are orders of magnitude smaller.

\section{RESULTS AND DISCUSSION}

For the mean seed injection energy range and geometrical sizes chosen, all the discharges modelled follow a similar evolution in the prebreakdown and breakdown phases (see Figs. 2, 3). As Fig. (2a) shows, the initial electric field across the gap does not penetrate very far into the hollow cathode backspace. Therefore, any particles present in this region are the result of ionization initiated by the seed electrons. Electrons (see Figs. 3,10a) travelling downstream eventually arrive at a place where the electric field causes the particles to accelerate and attain energies sufficient for ionization. Moving further downstream, these electrons feel a rapidly increasing electric field. Consequently, the ionization mean-free-path increases and they quickly travel to the anode without anymore collision events. Ions (see Fig. 3) move much more slowly than the electrons due to their much greater mass. This means as electrons are pulled to the anode an excess of ions are left behind in the neighbourhood of the cathode exit. Eventually, this region of positive charge, otherwise called the virtual anode, grows large enough to distort the electric field (compare Figs. $(\mathbf{2 b}, \mathbf{d})$ and $(\mathbf{3 b}, \mathbf{d}))$. The distortion simultaneously decreases the net voltage across the gap while growing, upstream accelerating electrons that are deeper in the hollow cavity to ionizing energies. Consequently, the ionization collision mean-freepath decreases in both regions as the virtual anode grows and the electron current arriving at the anode (see Fig. 4) continues to increase until the voltage drop across the gap approaches zero. Fig. (3) also demonstrates that collisional scattering results in a substantial spread of particles about the initial source injection direction placing electrons in positions where the electric field can accelerate them enhancing the two-dimensional growth of the virtual anode. 

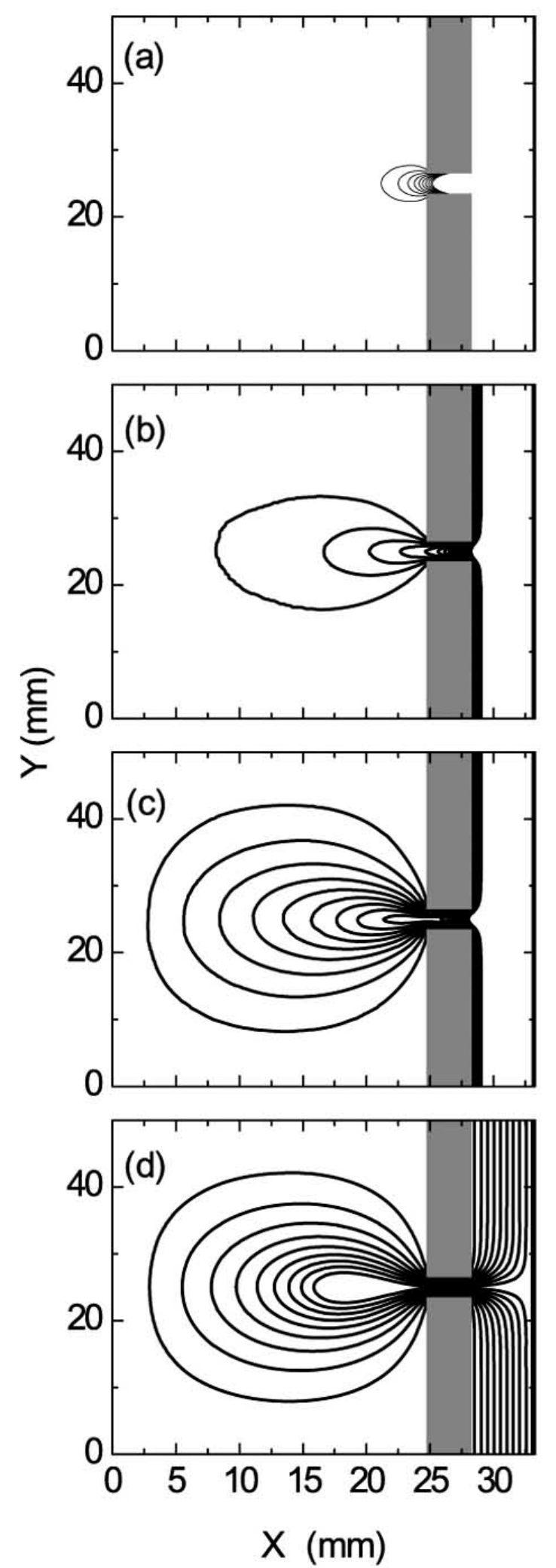

Fig. (2). The electrostatic potential, where (a) time $=0$, contour levels $=20 \mathrm{eV}(\mathbf{b})$ time $=46 \mathrm{~ns}$, contour levels $=100 \mathrm{eV}(\mathbf{c})$ time $=$ $52 \mathrm{~ns}$, contour levels $=100 \mathrm{eV},(\mathbf{d})$ time $=69 \mathrm{~ns}$ (peak current is achieved), contour levels $=1000 \mathrm{eV}$, and the first twenty contours are shown. The mean seed injection energy is $1 \mathrm{keV}$, and the neutral gas pressure is 0.18 torr.
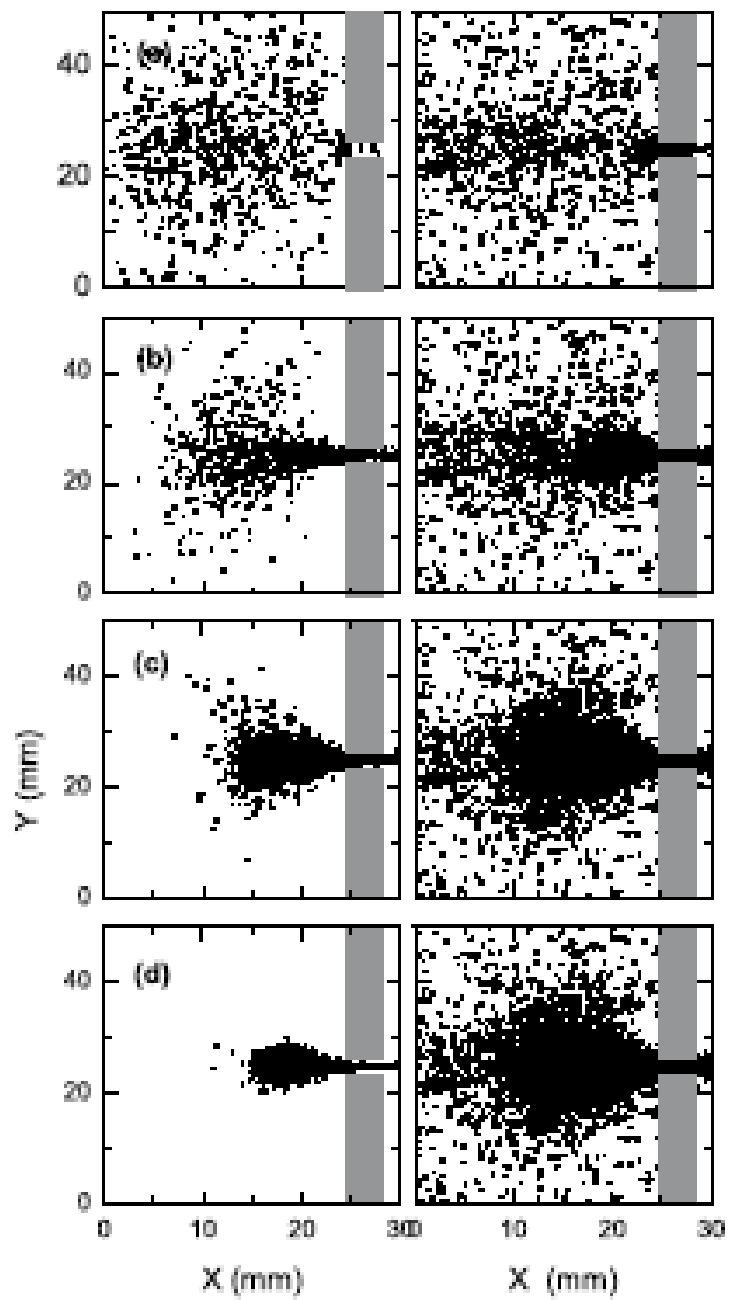

Fig. (3). Electron $X-Y$ space in the left column and Argon ions in the right at the following times (a) $t=15,($ b) $t=46 n s,(c) t=57 n s$ and (d) 69 ns (when the peak electron current is achieved). The mean seed injection energy is $1 \mathrm{keV}$, and the neutral gas pressure is 0.18 torr.

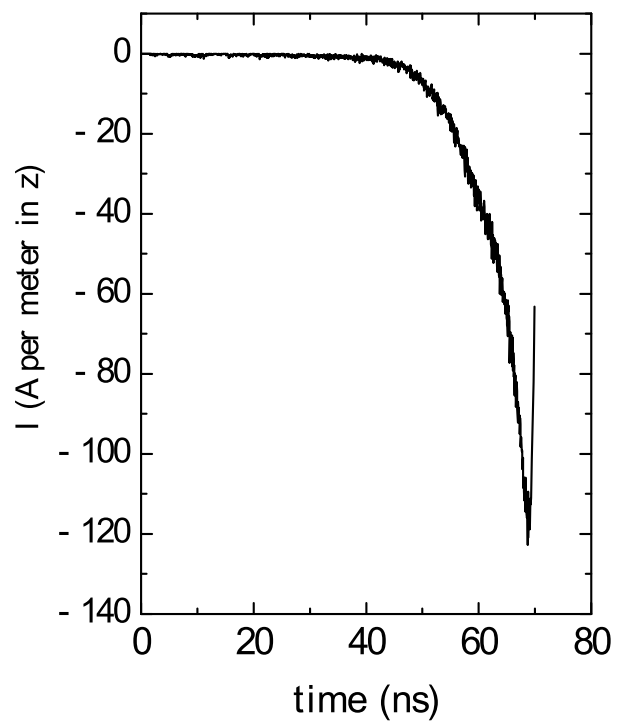

Fig. (4). The electron current arriving at the anode in the breakdown phase, where the peak value occurs at $69 \mathrm{~ns}$, the mean seed injection energy is $1 \mathrm{keV}$ and the neutral gas pressure is 0.18 torr. 
Examining the rate of development of the virtual anode brings to light the strong influence that the seed's mean injection speed has on this issue. Plots of the time to reach the peak electron current to the anode, $t_{p}$, (see Fig. 5) versus the neutral gas pressure, $\mathrm{P}$, for a range of mean seed kinetic energies was plotted. From the minima in these graphs, an optimum neutral gas pressure, $\mathrm{P}_{\min }$, which results in the fastest rate of growth can be determined for each mean injection energy. Figs. $(6,7)$ show these values of the neutral gas pressure and the time to the peak current, $t_{\min }$, as a function of the mean seed injection kinetic energy. Inspecting these plots reveals that for injection velocities substantially above that corresponding to the ionization peak cross-section $(\geq 300 \mathrm{eV})$ there are linear trends. There is an inverse dependence of $t_{\min }$ on the kinetic energy of the seed while $P_{\min }$ is proportional to this energy. The product of these two values, $\mathrm{P}_{\min } \mathrm{t}_{\min }$, is approximately constant in the energy range $300 \leq$ (kinetic energy) $\leq 900 \mathrm{eV}$ (see Fig. 8).

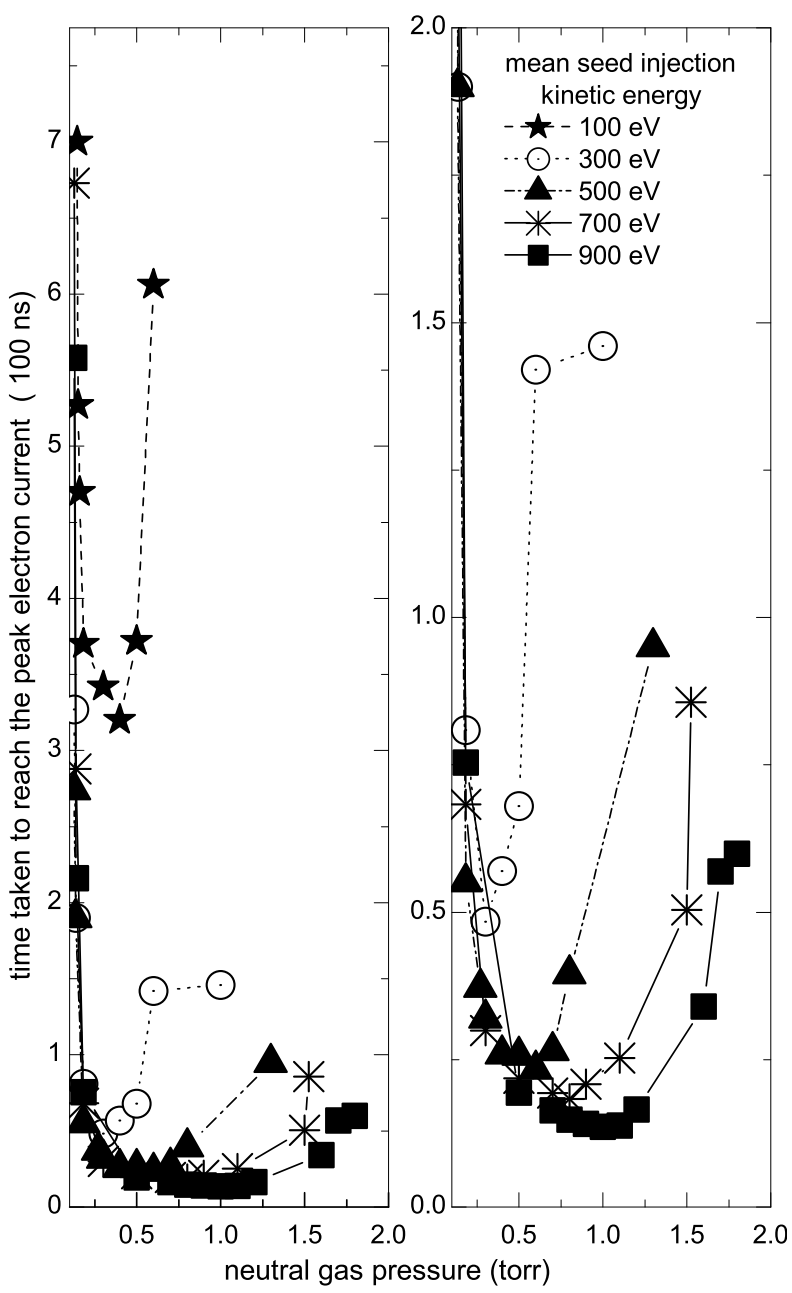

Fig. (5). The time taken to reach the electron peak current $t_{p}$ versus the neutral gas pressure $P$. The right side is a closer view of the minima for mean injection speeds above $100 \mathrm{eV}$.

A distinction can be drawn between the different seed energies used in the study, according to how the discharge develops in the first two phases of operation. Such a division of energies was noted in a previous investigation (a constant

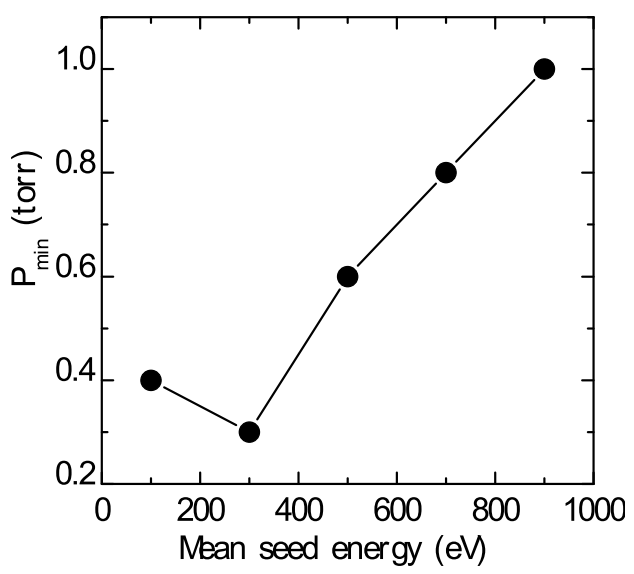

Fig. (6). The magnitude of the neutral gas pressure at the minimum of the $t_{p}$ versus $P$ curve, $P_{\min }$, against the mean seed injection energy.

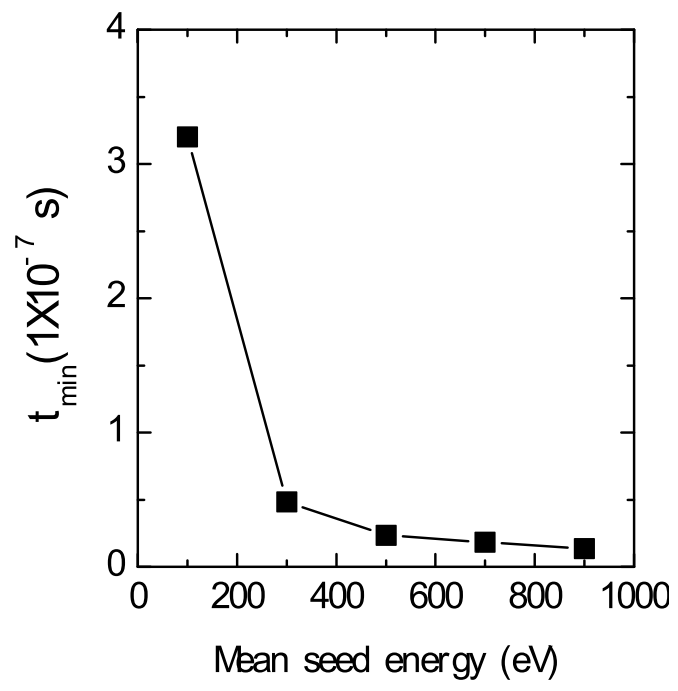

Fig. (7). The magnitude of the time to reach the electron peak current at the minimum of the $t_{p}$ versus $P$ curve, $t_{\min }$, against the mean seed injection energy.

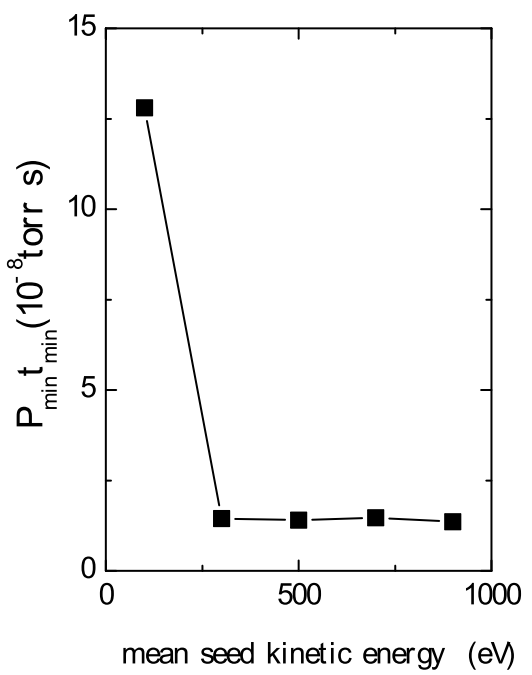

Fig. (8). The product of the neutral gas pressure at the fastest time to reach the peak current and this time versus the injection energy of the electrons. 
neutral gas pressure was used) [17], where it was shown that the spatial and temporal development of the discharge varies with the mean kinetic energy range of the seed electrons. The seed was injected in the same location as the current study. In the low energy range, where the mean seed energy is insufficient to ionize the neutral gas, the electrons were shown to travel relatively slowly to the exit, prolonging the onset of an avalanche in this region. Injecting seed electrons in the mid-energy range at a value corresponding to the peak in the ionization cross-section resulted in an initial avalanche in front of the injection point. These additional electrons, which also moved slowly downstream at energies less than the ionization threshold, resulted in a more rapid growth of the virtual anode. Also, high energy seed electrons were injected with mean kinetic energies substantially above that corresponding to the peak ionization cross-section. As these faster electrons travel downstream each collision energy loss increases the ionization cross-section resulting in a much quicker onset of an avalanche near the cathode exit compared to the other energy ranges.

Figs. $(6,9)$ demonstrate a clear difference between the discharge properties with the mean electron injection energies. Fig. (6) of the neutral gas pressure at the minimum in the $t_{p}$ versus $P$ plots, $P_{\text {min }}$, plotted against the mean seed kinetic energy has a minimum at a seed energy of $300 \mathrm{eV}$ while the graph of the mean-free-path as a function of the electron energy (see Fig. 9) has a maximum at this value. The latter graph was calculated using the density at $\mathrm{P}_{\min }$ in conjunction with the ionization cross-section at a value of the average kinetic energy of the seed. These figures crudely illustrate a separation between the case of initial injection speeds that results in decreasing ionization cross-sections as the electrons travel downstream and the opposite situation of increasing collision probabilities. The value of the seed energy at the minima and maxima in these graphs should not be interpreted as an exact boundary. That would require closer look using a more refined scale in this neighbourhood, which is outside of the intended scope of this paper.

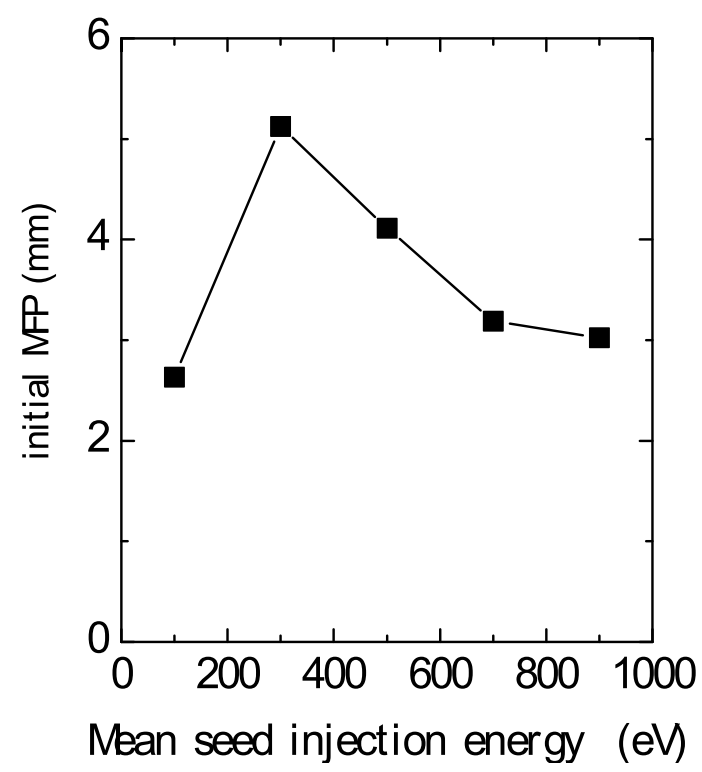

Fig. (9). The initial ionization mean-free-path (MFP) resulting from the inverse product of the neutral gas density at $\mathrm{P}_{\min }$ and the crosssection at the mean injection energy of the seed electrons.
On the left-hand-side of the $t_{p}$ versus $P$ curves in Fig. (5) the time increases without bound indicating the minimum neutral gas pressure required for the discharge to achieve breakdown. There are also limits in how far the neutral gas pressure can be increased on the right-hand side of the curve before the formation of a pseudospark discharge is inhibited. Substantial increases in the pressure results in the formation of a plasma primarily confined within the anode-cathode gap, as Fig. (10) illustrates. Upon leaving the source the electrons rapidly lose their mean velocity due to ionization energy losses. It is the avalanche that ensues after a few of these electrons arrive in the region where the initial penetrating electric field can accelerate them to ionizing energies again that dominates the plasma generation.

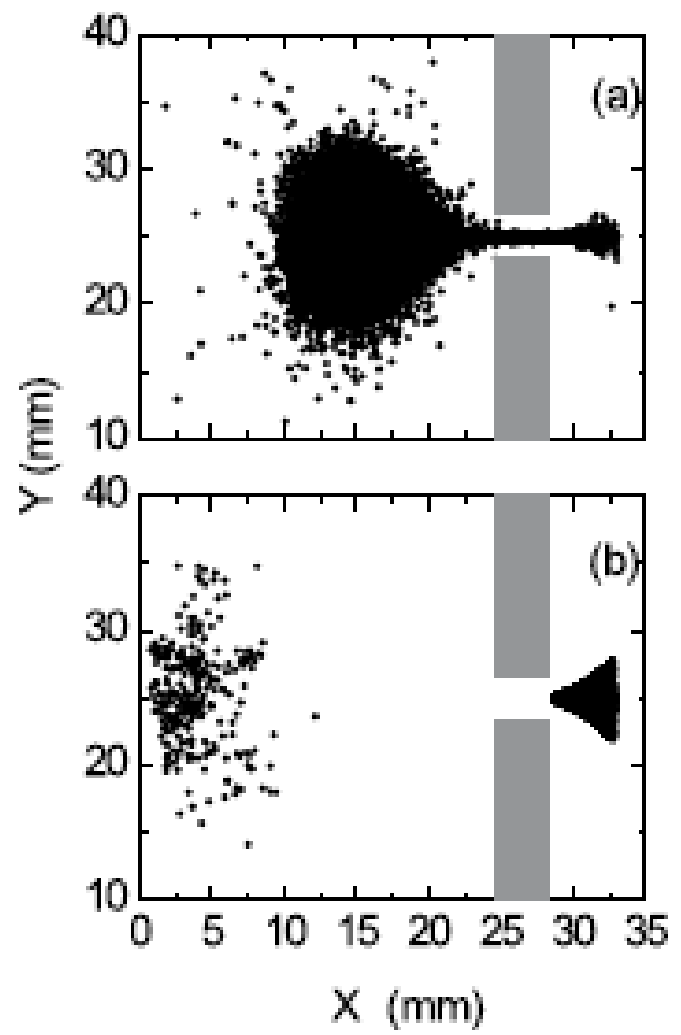

Fig. (10). Electron $X-Y$ phase space at the time of the peak electron current where the mean seed injection energy is $500 \mathrm{eV}$ and the neutral gas pressure is (a) 0.127 and (b) 1.5 torr.

\section{CONCLUSIONS AND SUMMARY}

Development of the psuedospark discharge in the prebreakdown phase is shown to vary with the mean kinetic energy of the seed electrons. Two main energy ranges are identified; 1, mid-energy, around values that result in a decrease in the ionization cross-section after collision energy losses and (2) high-energy, where the cross-section increases after each collision event, resulting in the early formation of a plasma (a virtual anode) downstream near the exit. This means, in the latter case, that a neutral gas pressure that permits or enhances this early migration gives a faster delay time. Across both energy ranges, the neutral gas pressure that produces the fastest delay time for a given average seed electron injection energy is shown to vary. Furthermore, for seed injection speeds that fall into the high energy range, the product of the fastest delay time and the pressure at which this occurs over the different seed energies is constant. 


\section{ACKNOWLEDGEMENTS}

This work was supported by the Office of the Secretary of Defence under grant No. FA8650-04-C-2511, and by the U.S. DOE Office of Science, Office of High Energy Physics under Grant No. DE-FG03-99ER82903.

\section{REFERENCES}

[1] Tkotz R, Gortler A, Christiansen J, et al. Pseudospark switchestechnological aspects and application. IEEE Trans Plasma Sci 1995; 23: 309- 17

[2] Choi P Chuaqui $\mathrm{H}$, Lunney J, et al. Plasma formation in a pseudospark discharge. IEEE Trans Plasma Sci 1989; 17: 770-4.

[3] Frank K, Boggasch E, Christiansen J, et al. High-power pseudospark and BLT switches. IEEE Trans Plasma Sci 1988; 16: 317-23.

[4] Pitchford LC, Ouadoudi N, Boeuf JP, et al. Triggered breakdown in low-pressure hollow cathode (pseudospark) discharges. J Appl Phys 1995; 78: 77-90.

[5] Gastel M, Hillmann H, Muller F, Westheide J. Influence of the hollow cathode dimensions on the electron beam current in a pseudospark discharge. IEEE Trans Plasma Sci 1995; 23: 248-53.

[6] Frank K, Christiansen J. The fundamentals of the pseudospark and its applications IEEE Trans Plasma Sci 1989; 17: 748-53.

[7] Jain KK, Ding BN, Rhee MJ. Scaling study of pseudospark produced electron beam: IEEE Particle Accelerator Conference. Conference Proceedings. San Francisco, CA, May 6-9, 1991; pp. 1972-4.

[8] Mehr T, Arenz H, Bickel P, et al. Trigger devices for pseudospark switches. IEEE Trans Plasma Sci 1995; 23: 324-29.

[9] Iberler M, Bischoff R, Frank K, et al. Fundamental investigation in two flashover-based trigger methods for low-pressure gas discharge switches. IEEE Trans Plasma Sci 2004; 32: 208-14.

[10] Legentil M, Postel C, Thomaz Jr. JC, Puech V. Corona-plasma triggered pseudospark discharges. IEEE Trans Plasma Sci 1995; 23: $330-4$

[11] Korolev YD, Geyman VG, Frants OB, et al. Low-voltage triggering for a pseudospark switch with an auxiliary glow discharge IEEE Trans Plasma Sci 2001; 29: 796-801.

[12] Dwivedi HK, Urban J, Frank K. Role of trigger to avoid current quenching in pseudospark switch. IEEE Trans Plasma Sci 2002; 30: 1371-5.

[13] Bloess D, Kamber I, Riege H, et al. The triggered pseudo-spark chamber as a fast switch and as a high-intensity beam source. Nucl Instr Methods 1983; 205: 173-84

[14] Zambra M, Moreno J, Inostroza J, Juan C, Araneda JC. Experimental observations of the virtual anode motion and streamer breakdown mechanisms in a pseudospark discharge. IEEE Trans Plasma Sci 2004; 32: 221-6.

[15] Lin B, Chow Q. Breakdown voltages of pseudospark in He, Ar, and $\mathrm{N}_{2}$. IEEE Trans Plasma Sci 1995; 23: 239-42.

[16] Anders A, Anders S, Gundersen MA, Martsinovskii AM. Selfsustained self-sputtering: a possible mechanism for the superdense glow phase of a pseudospark. IEEE Trans Plasma Sci 1995; 23: 275-82.

[17] Cetiner SO, Stoltz P, Messmer P, Cambier J-L. Dependence of electron peak current on hollow cathode dimensions and seed electron energy in a pseudospark discharge. J Appl Phys 2008; 103 : 023304-1-9.

[18] Sato N, Sakamoto S. Undervoltage breakdown between parallel plates in air. J Phys D: Appl Phys 1979; 12: 875-85.

[19] Cooley JE, Choueiri EY. Fundamentals of PPT discharge initiation: undervoltage breakdown through electron pulse injection. 39th
AIAA Joint Propulsion Conference. Huntsville, AL, July 20-23, 2003; pp. 1-11.

[20] Cooley JE, Choueiri EY. Fundamentals of discharge initiation in gas-fed pulsed plasma thrusters. $29^{\text {th }}$ International Electric Propulsion Conference. Princeton University, Oct $31^{\text {st }}-\mathrm{Nov} 4^{\text {th }}$, 2005; pp. 1-11.

[21] Verboncoeur JP, Langden AB, Gladd NT. An object-oriented electromagnetic PIC code. Comput Phys Commun 1995; 87: 199211.

[22] Vahedi V, Surendra M. A Monte Carlo collision model for the particle-in-cell method: applications to argon and oxygen discharges. Comput Phys Commun 1995; 87: 179-98.

[23] Bruhwiler DL, Giacone RE, Cary JR, et al. Particle-in-cell simulations of plasma accelerators and electron-neutral collisions. Phys Rev Special Top Accel Beams 2001; 4: 101302-1-13.

[24] Younger SM, Märk TD. Electron impact ionization. Märk TD, Dunn GH, Eds. Vienna: Springer Verlag 1985; pp. 24-41.

[25] Brown SC. Basic data of plasma physics in the fundamental data on electrical discharges in gases. New York: American Institute of Physics 1994.

[26] Jackson JD. Classical electrodynamics. New York: Wiley 1975; pp. 618-53.

[27] Rieke FF, Prepejchal W. Ionization cross sections of gaseous atoms and molecules for high-energy electrons and positrons. Phys Rev A 1972; 6: 1507-19.

[28] Perkins ST. LLNL report UCRL-50400 1991; 31: unpublished.

[29] Fermi E. The ionization loss of energy in gases and in condensed materials. Phys Rev 1940; 57: 485-93.

[30] Rudd ME. Differential and total cross sections for ionization of helium and hydrogen by electrons. Phys Rev A 1991; 44: 1644-52.

[31] Rudd ME, Hollman KW, Lewis JK, Johnson DL, Porter RR, Faherquist EL. Doubly differential electron-production cross sections for 200-1500-eV $e^{-}+\mathrm{H}_{2}$ collisions. Phys Rev A 1993; 47: 1866-73.

[32] Rossi B. High-energy particles. New York: Prentice Hall 1952; pp. 27-9.

[33] Reiser M. Theory and design of charged particle beams. New York: Wiley 1994; pp. 273-8.

[34] Younger SM. Electron impact ionization cross sections and rates for highly ionized atoms. J Quant Spectrosc Radiat Transf 1981; 26: 329-37.

[35] Bray I, Fursa DV, McCarthy IE. Calculation of electron-lithium scattering using the coupled-channel optical method. Phys Rev A 1993; 47: 1101-10

[36] Opal CB, Peterson WK, Beatty EC. Measurements of secondaryelectron spectra produced by electron impact ionization of a number of simple gases. J Chem Phys 1971; 55: 4100-7.

[37] Rudd ME. Differential and total cross sections for ionization of helium and hydrogen by electrons. Phys Rev A 1991; 44: 1644-52.

[38] Rudd ME, Hollman KW, Lewis JK, Johnson DL, Porter RR, Fagerquist EL. Doubly differential electron-production cross sections for 200-1500-eV $e^{-}+\mathrm{H}_{2}$ collisions. Phys Rev A 1993; 47: 1866-73.

[39] Slinker SP, Taylor RD, Ali AW. Electron energy deposition in atomic oxygen. J Appl Phys 1988; 63: 1-10.

[40] Bethe HA, Ashkin J. Passage of radiations through matter. experimental nuclear physics. New York: Wiley \& Sons 1953; vol. 1: pp. 252-304.

[41] Mott NF, Massey HSW. The theory of atomic collisions. $2^{\text {nd }}$ ed. Oxford: Oxford University Press 1949.

[42] Goldstein H. Classical mechanics. Reading: Addison-Wesley 1980

[43] Perkins ST. LLNL report UCRL-50400, 1991; unpublished. 